\title{
Numerical Variational Methods for Data Filtering and Interpolation
}

\author{
Christine Potier AND Christine VerCKen \\ Département Informatique, Ecole Nationale Supérieure des Télécommunications, Paris, France
}

(Manuscript received 14 August 1984, in final form 8 April 1985)

\begin{abstract}
An objective representation of an observed meteorological field is obtained by minimizing a quadratic functional that measures both the smoothness (and regularity) of the objective field and the closeness to the observed data. This is a particular form of the general model for numerical variational analysis suggested by Wahba and Wendelberger to generalize the idea introduced by Sasaki.

The solution of this minimization problem can be obtained by using homogeneous splines and cross validation or by using the finite element method to determine an approximate solution. Finite elements can provide data compression for large $N$. Testud and Chong proposed such a method, based on bilinear finite elements for 3dimensional wind field analysis. We go further with the same principle and study a more regular approximation, which can be analytically differentiated, obtained by bicubic splines.

The numerical simulations proposed by Chong and Testud are used to compare the capability of all these methods (splines, bilinear elements, and bicubic splines) to restitute the wind field and its divergence.

The finite elements give quite accurate results, especially the bicubic splines, and are very easy to compute.
\end{abstract}

\section{Numerical variational objective analysis}

\section{a. Introduction}

The objective analysis of a meteorological field consists in filtering and interpolating measurement data in order to reconstruct the field objectively, or at least in specifying its values at the nodes of a regular grid. The objective field must satisfy an "observational constraint" (minimizing the variance between the observed and analyzed field), while respecting certain physical constraints, for example, balance equations, and the need for regularity. Thus, the objective field can be sought as the solution to a general variational problem: given a functional $I$, determine the function that minimizes $I$ [ subject to some exact (strong) or approximate (weak) constraints].

Sasaki (1971) introduced numerical variational methods for objective analysis. Given the observed field $\tilde{u}(x, y, t)$, the objective field is the minimizer $u$ of

$$
\begin{aligned}
I(u)= & \int\left\{a_{1}(u-\tilde{u})^{2}+a_{2}\left(\frac{\partial u}{\partial t}+c_{x} \frac{\partial u}{\partial x}+c_{y} \frac{\partial u}{\partial y}\right)^{2}\right. \\
& \left.+a_{t}\left(\frac{\partial u}{\partial t}\right)^{2}+a_{h}\left[\left(\frac{\partial u}{\partial x}\right)^{2}+\left(\frac{\partial u}{\partial y}\right)^{2}\right]\right\} d x d y d t .
\end{aligned}
$$

The first term is the observational constraint; the second term is a weak constraint of advection; the last terms, which are low-pass filters of frequency and wavenumbers, eliminate possible discontinuities of the analyzed field.

After some simplifying assumptions, Sasaki obtained the Euler equation of this variational problem (1.1), that is, an elliptic second-order partial differential equation. When the parameters $a$ are specified, this equation can be solved numerically after the discrete data have been interpolated on a fine grid representing numerical discretization of a continuous function. This formulation allowed Sasaki to compare the weighting factors of conventional objective analysis (Cressman, Endlich and Mancuso, Inman, etc.) to Green's function $G$ (or fundamental solution) of the differential operator associated to the Euler equation of (1.1). If $G$ is known, then the solution $u$ is

$$
\begin{aligned}
u(x, y, t)=\int G(x, y, t ; & \left.x^{\prime}, y^{\prime}, t^{\prime}\right) \\
& \times k \tilde{u}\left(x^{\prime}, y^{\prime}, t^{\prime}\right) d x^{\prime} d y^{\prime} d t^{\prime} .
\end{aligned}
$$

Sasaki obtained an unbounded Green's function. He was thus led to obtain the solution of Euler equation while using finite differences method. Green's function was then used to validate the empirical weighting factors.

So as to avoid the inconvenience of two-staged data processing (interpolation on a fine grid, and then numerical solution of a partial differential equation with boundary conditions), Wahba and Wendelberger (1980) then proposed to consider the observational constraint at the original data points and to minimize the functional directly:

$$
I(u)=1 / N \sum_{i=1}^{N}\left[u\left(t_{i}\right)-z_{i}\right]^{2}+\mu J_{m}(u)
$$


where

$$
J_{m}(u)=\sum_{\alpha_{1}+\cdots+\alpha_{d}=m} \frac{m !}{\alpha_{1} ! \cdots \alpha_{d} !} \int_{\Omega}\left(\frac{\partial^{m} u}{\partial x_{1}^{\alpha_{1}} \cdots \partial \chi_{d}^{\alpha_{d}}}\right)^{2}
$$

or a similar functional, to take into account the advection or any other weak constraint. This is the general form that we will use thereafter; the parameter $m$ reflects the known regularity of our field.

\section{b. Aspects of the minimization problem}

Generally speaking, let the analyzed field depend upon $d$ variables ( $d=2,3$, or even more) and be defined upon a domain $\Omega$ of $R^{d}$, and the functional $I$ be defined as in (1.3).

The parameters $m$ and $\mu$ specified, the minimizer $u_{m \mu}$ of $I$ is sought in the space $V$ of all the admissible functions for $I$ : continuous functions for which all partial derivatives (in the distributional sense) of total order $m$ are square integrable upon $\Omega$. Writing the Euler equation of (1.3), it can be shown that the minimizer $u$ of $I$ verifies $\Delta^{m} u=0$ in the interior of $\Omega$, except at the points $\left\{t_{i}\right\}, i=1$ to $N$.

Provided that $m>d / 2$ (in this case, $V$ is a space of continuous function, as the fundamental solution of $m$-iterated Laplacian $\Delta^{m}$ is continuous), and that there is a $P_{m-1}$ unisolvent subset of $\left\{t_{i}\right\}_{i=1}{ }^{N}$ (every polynomial of degree $\leqslant m-1$, null on this subset, is the null polynomial), the minimizer $u_{m \mu}$ of (1.3) exists, is unique in $V$, and depends continuously upon the data.

The choice of the domain $\Omega$ is very important for the numerical determination of the solution:

- If $\Omega$ is the whole space $R^{d}$, the solution is known analytically, being a multivariate Dm-spline, and its coefficients can be obtained without any additional discretization by solving a linear system approximately of dimension $N$.

- If the domain is a bounded subset, the minimization of $I$ is formally equivalent to a $2 m$-order problem with natural boundary conditions, and the solution will depend on the geometry of $\Omega$. However, we can easily determine an approximate solution to the variational problem in a $n$-dimensional subspace of $V$ by using the finite element method.

\section{c. Dm-splines}

A general mathematical theory of multivariate homogeneous (invariant by translation and rotation) splines was shown by Duchon (1977) and further studied by Meinguet (1979) and Wahba (1979).

For $m>d / 2$, a Dm-spline $u$ is a sum of $N$ fundamental solutions of $\Delta^{m}$ in $R^{d}$, plus a polynomial $p$ of degree $m-1$ :

$$
u(t)=\sum_{i=1}^{N} a_{i} K_{m}\left(t, t_{i}\right)+\sum_{j=1}^{M} b_{j} p_{j}(t)
$$

where $p_{1}, \ldots, p_{M}$ is a basis of $P_{m-1}$, the space of polynomials of $d$ variables with degree strictly less than $m$, which is of dimension

$$
M=\left(\begin{array}{c}
m-1+d \\
d
\end{array}\right)
$$

The Dm-spline is well defined if there is a subset $\left\{t_{i_{1}}\right.$, $\left.\ldots, t_{i M}\right\}$ such that any polynomial $p \in P_{m-1}$ is uniquely defined by its values on this subset.

The functions $K_{m}\left(t, t_{i}\right)$ depend only on $\left|t-t_{i}\right|=r$ :

$$
K_{m}\left(t, t_{i}\right)=\left\{\begin{array}{ll}
r^{2 m-d} \log r & \text { if } d \text { is even } \\
r^{2 m-d} & \text { if } d \text { is odd }
\end{array} .\right.
$$

The minimizer of $I(u)$ is the Dm-spline solution of

$$
\left.\begin{array}{c}
\left(C_{m d} N \mu\right) a_{i}+\sum_{j=1}^{N} K_{m}\left(t_{i}, t_{j}\right) a_{j}+\sum_{k=1}^{M} b_{k} p_{k}\left(t_{i}\right)=z_{i} \\
i=1 \quad \text { to } N \\
\sum_{j=1}^{N} a_{j} p_{k}\left(t_{j}\right)=0, \quad k=1 \quad \text { to } M
\end{array}\right\}
$$

in which the constant $C_{m d}$ (see Wahba, 1979) appears because $K_{m}\left(t, t^{\prime}\right)$ is only proportional to the fundamental solution of $\Delta^{m}$, when the parameter $\mu$ is considered as a physical filtering parameter. When $\mu=0$, we obtain the interpolating spline, and if $\mu$ tends toward infinity, the splines approach the least squares polynomial (Harder and Desmarais, 1972).

To determine numerically an interpolating or a smoothing Dm-spline, it is necessary to solve a linear system of $N+M$ equations. The matrix $\mathbf{A}$ is a full matrix, and the system can be solved by using Gaussian elimination if $N$ is not too large. Meinguet (1979) studied some transformations of the original basis $\left[K_{m}\left(t_{i}\right.\right.$, $\left.t_{j}\right)$ ] that enable one to obtain the solution in the following way. First, one must solve a system of $N-M$ linear equations whose coefficient matrix is symmetric and positive definite, by using LU or Choleski factorization; later, solve two $M$-dimensional systems. However, as the run-time increases by $N^{3}$, and since the required storage increases by $N^{2}$, it is necessary to use a method proposed by Utreras (1979) when there is a large amount of data. Utreras proposed beginning with piecewise calculations on nondisjoint subsets, and then fitting the pieces together by using a continuously differentiable weighting function.

The choice of the parameter $m$ is important and must reflect the known regularity of the true field $f$. If $f$ and all its derivatives up to order $m$ are square integrable, then $f$ and all its derivatives up to order $s(s$ being an integer verifying $s<m-d / 2$ ) are continuous. Their estimation by the Dm-spline is thus pointwise convergent in a bounded subset $\Omega$, when the set $\left\{t_{i}\right\}_{i=1}^{N}$ becomes dense in $\Omega$. For example, "thin-plate" splines ( $m=2$ and $d=2$ ) are continuously differen- 
tiable, but should not, in theory, be used to estimate derivatives. It is mathematically more correct for derivative estimation, to choose $m \geqslant 3$, or to use a more general spline, the "pseudocubic"

$$
u(t)=\sum_{i=1}^{N} a_{i}\left|t-t_{i}\right|^{3}+b x+c y+d .
$$

This is as simple as the "thin-plate" spline, although it is obtained by minimizing a complicated quadratic functional involving Fourier transforms. However, in our numerical simulations, the approximation of the function and its derivatives obtained by using "thinplate" or "pseudocubic" are quite similar.

Smoothing may be viewed as applying a low-pass filter to the data. The parameter $\mu$ controls the "cutoff wavenumber", while $m$ controls the steepness of the roll-off. Wahba and Wendelberger (1980) propose a very elaborate cross-validation method to estimate optimum $\mu$ and $m$ from the data being analyzed: GCV. The GCV method estimates $\mu$ and $m$ for which the implied data filter has maximum internal predictive ability. This is done by leaving out one data point at a time and by determining how well the missing datum can be predicted from the remaining data. Wahba and Wendelberger's numerical algorithm determines the optimum $\mu$ ( $m$ being fixed), and when this has been done for a few values of $m$ the optimum $(m, \mu)$ can be selected. However, for every $m$, this algorithm leads to an eigenvalue problem of dimension $N-M$, and it is necessary to determine all eigenvalues.

\section{d. Finite element method}

The number of coefficients of an interpolating or a smoothing spline is almost equal to the number of data. When there is a large amount of data, this kind of approximation is extremely consuming of computer resources and sometimes it is necessary to reduce the size of the approximation. This is impossible when interpolating, as each equality constraint represents a degree of freedom, and we have to do piecewise interpolation. But, when there is only weak constraints (to be satisfied approximately), we can seek the minimizer on a finite dimensional subspace $V_{n}$ of $V$, with dimension $n$ much smaller than $N$. For example, if

$$
I(v)=\sum_{i=1}^{N}\left[v\left(t_{i}\right)-z_{i}\right]^{2}
$$

this method is the classical least-squares fit.

Let $w_{1}, \cdots w_{n}$ be a basis of $V_{n}$. Substituting

$$
v=\sum_{i=1}^{n} a_{i} w_{i}
$$

into (1.3) and requiring that the derivatives of $I$ with respect to the coefficients $a_{i}$ must vanish, yields the following system of equations:

$$
\sum_{j=1}^{N} a_{j} m_{i j}=f_{i} \text { for } i=1 \text { to } n
$$

where

$$
\begin{aligned}
m_{i j} & =1 / N \sum_{k=1}^{N} w_{i}\left(t_{k}\right) w_{j}\left(t_{k}\right)+\mu\left(w_{i}, w_{j}\right)_{m}, \\
\left(w_{i}, w_{j}\right)_{m} & =\sum_{\alpha_{1}+\alpha_{2}=m} \frac{m !}{\alpha_{1} ! \alpha_{2} !} \int_{\Omega}\left(\frac{\partial^{m} w_{i}}{\partial x^{\alpha_{1}} \partial y^{\alpha_{2}}}\right)\left(\frac{\partial^{m} w_{j}}{\partial x^{\alpha_{1}} \partial y^{\alpha_{2}}}\right)
\end{aligned}
$$

and

$$
f_{i}=1 / N \sum_{k=1}^{N} z_{k} w_{i}\left(t_{k}\right)
$$

or in matrix notation

$$
\mathbf{M} a=f
$$

with $\boldsymbol{M}$ called the energy matrix.

The parameter $\mu$ can be estimated by using the GCV method. In this case, as pointed out by Bates and Wahba (1983), it requires the calculation of the same number $n$ of values in a singular value decomposition as the dimension $n$ of the subspace $V_{n}$.

The accuracy of the approximation depends on the choice of the subspace $V_{n}$, a choice that is made with the following considerations:

- The approximation in $V_{n}$ must converge towards the exact solution as $n$ tends to infinity, for the approximation to be consistent.

- It must be easy to compute and numerically stable: the functions in $V_{n}$ must be simple, and there must be a local basis so that the discrete approximation is the solution of a sparse system.

The basic finite element method is characterized by three aspects, that for simplicity, we have described when $\Omega$ is a bounded convex domain of the plane.

- There is a subdivision $T_{h}$ of $\Omega$ into triangles or rectangles in such a way as two elements of $T_{h}$ have nothing in common, or just a vertex or a whole edge. Then $\Omega$ (or nearly $\Omega$ if its boundary is not polygonal) is written as a finite union of finite elements, the subsets $K \in T_{h}$.

- The subspace $V_{n}$ is a space of piecewise polynomial functions on the finite elements: their restriction to each $K \in T_{h}$ is a polynomial.

- The functions $v$ are easily determined by a simple set of nodal values. (There is a set of basis functions with small supports that depend on the subdivision $T_{h}$.) Nevertheless, they have the desired degree of continuity: $(m-1)$ times continuously differentiable for the minimization of a functional of order $m$.

The dimension $n$ of $V_{n}$ depends on the number of vertices of the subdivision and on the number of nodal values necessary at each vertex. The accuracy of the approximation depends on the step $h$ (the length of the largest edge in the subdivision) and on the degree of piecewise polynomials. The dimension $n$ thus reflects the discretization. 
Triangular elements are very useful for boundary value problems (Strang and Fix, 1973) because they are better than rectangles at approximating a curve boundary, but rectangles permit more simple elements of high degree. Finite elements admissible for $J_{m}$ must be $(m-1)$ times continuously differentiable. As the smoothing problem (1.3) is well defined in the plane only when $m>1$, we must use finite elements of at least class $C^{1}$. The most simple, continuously differentiable, triangular element is called the Argyris quintic element (Strang and Fix, 1973). On each triangle, the values of the function and all its derivatives up to the second order at the three nodes are necessary to determine the polynomial. The complexity of functions of $V_{n}$ grows very fast when one looks for greater continuity. Moreover, it is very hard to extend when the domain $\Omega$ is in $R^{d}, d \geqslant 3$.

As in the smoothing problem there are no boundary conditions; the domain $\Omega$ can be taken as rectangular, containing all the data points and the domain of interest.

\section{e. Rectangular elements}

Now let $\Omega$ be a rectangle $[a, b] \times[c, d], a=x_{1}$ $<x_{2} \cdots<x_{r}=b$ be a subdivision of $[a, b]$ and $c=y_{1}$ $<\cdots<y_{s}=d$ a subdivision of $[c, d]$.

The simplest construction is based on functions that are bilinear in each rectangle $d_{i j}=\left[x_{i}, x_{i+1}\right] \times\left[y_{j}, y_{j+1}\right]$. On a rectangle the polynomial is determined by the values of $v$ at each node. The function is thus only continuous on the whole domain $\Omega$ and is in theory not admissible for $J_{m}$. However, Testud and Chong (1983) used this element successfully to find an approximate solution to the problem (1.3).

For $C^{1}$ continuity, the Hermite bicubic element is very simple. It is the natural extension of the Hermite cubic in one dimension; over each interval a cubic polynomial is determined by the values of the function and its first derivative at the two end points. The Hermite bicubic is a product of two such spaces, and on a rectangle the bicubic is determined by the values of $v, d v / d x, d v / d y, d^{2} v / d x d y$ at each node; the function is thus continuously differentiable on the whole domain $\Omega$.

The Hermite bicubic can easily be extended so as to increase continuity up to order $m-1$. On each rectangle, the element is a product of polynomials of degree $2 m-1$ in each variable and is determined by $m^{2}$ values at each node. This element can also be extended to $R^{d}$. They are $m^{d}$ unknowns at each node and the construction becomes rapidly inefficient. The number of unknowns can be reduced by imposing additional continuity constraints. The space $S$ of bicubic splines with knots $\left\{x_{i}, y_{j}\right\}_{i=1}^{j=1}$ to io is defined by: i) on any rectangle $d_{i j}$ for $i=1$ to $r-1$ and $j=1$ to $s-1, s(x, y)$ is a bicubic polynomial; and ii) for $0 \leqslant i \leqslant 2$ and $0 \leqslant j$ $\leqslant 2$ the derivatives $\partial^{i+j} s(x, y) / \partial x^{i} \partial y^{j}$ are continuous on $\Omega$.
The space of bicubic splines is a subspace of Hermite bicubics, in which functions are twice differentiable and are determined by a single value at each node. They are as continuous as possible without becoming a single polynomial over the whole domain.

The dimension of $S$ is $n=(r+2)(s+2)$. There is a very simple basis that is a product of spline functions of minimal support: the cubic B-splines. Cubic splines cannot have a strictly local basis and each B-spline is nonzero only over four adjacent intervals.

Any element $s \in S$ can be written in this basis:

$$
s(x, y)=\sum_{i=-2}^{r-1} \sum_{j=-2}^{s-1} a_{i j} B_{i}(x) B_{j}(y) .
$$

To define the bicubic B-spline basis, $(r+6)(s+6)$ knots are necessary. It is usual to add three initial and three final arbitrary knots in each direction, which do not affect the value of the spline on the domain $\Omega$.

Appendix A provides a more detailed description of B-splines, and Appendix B shows how to calculate the solution of the minimization problem (1.3) in the subspace spanned by products of B-splines.

It must be noted, however, that although all these spaces are spanned by products of one-dimensional basis functions, the matrix $\boldsymbol{M}(1.13)$ of inner-product energy cannot be decomposed into one-dimensional operators because separation of variables cannot be applied to the quadratic rotation-invariant $I(v)$.

\section{Numerical results in simulated wind field analysis}

\section{a. Framework of the experience}

Let us recall the experience described by Testud and Chong (1983). A dual-radar system operates following the coplane methodology. The two radars $-R_{1}$ and $R_{2}$-scan simultaneously a common $\alpha$-plane that contains $R_{1} R_{2}$ axis and that is inclined at an angle $\alpha$ with respect to the horizontal plane. Within each $\alpha$-plane, the measured radial velocities $v_{1}$ and $v_{2}$ from $R_{1}$ and $R_{2}$, respectively, and reflectivity factors $z_{1}$ and $z_{2}$ must be used to estimate the two components $\Gamma$ and $\Psi$ of air motion:

$$
\left.\begin{array}{rl}
\Gamma(x, y)= & \left(r_{1} v_{1}-r_{2} v_{2}\right) / 2 D \\
\Psi(x, y)= & {\left[r_{2}(x+D) v_{2}\right.} \\
& \left.-r_{1}(x-D) v_{1}\right] / 2 y D+w_{t} \sin \alpha
\end{array}\right\},
$$

where $2 D$ corresponds to the distance between the two radars, $r_{1}=\left[(x+D)^{2}+y^{2}\right]^{1 / 2}$ and $r_{2}=\left[(x-D)^{2}\right.$ $\left.+y^{2}\right]^{1 / 2}$ and $w_{t}$ the terminal fall velocity of precipitation. This final parameter is estimated by an empirical relationship involving local reflectivity factor and the scale height of air density decrease.

Since the raw data are noisy, and the sampling of the two radars do not coincide within an $\alpha$-plane, the data have to be filtered and interpolated, at least on a common grid, so as to recover estimates of $\Gamma$ and $\Psi$. In addition, these estimates must be stable with respect 
to differentiation, because the recovery of the third component of the air motion (component perpendicular to the $\alpha$-plane) involves the integration of the continuity equation. An accurate estimation of the field and its divergence within each $\alpha$-plane is necessary.

Each field parameter $v_{1}, v_{2}, z_{1}, z_{2}$ will be estimated separately, and in the following, $u$ will stand for any of them, the measurement data being $\left\{\tilde{u}_{i}\right\}_{i=1}{ }^{N}$. We seek $u$ as the solution to the variational problem:

$$
\min I(v)=\frac{1}{N} \sum_{i=1}^{N}\left[v\left(t_{i}\right)-\tilde{u}_{i}\right]^{2}+\mu J_{m}(v)
$$

The solution will thus be the output of a low-pass filter that should reduce both the white noise due to the two radars and the turbulent motions with "intermediate" scales.

\section{b. Numerical test procedures}

The simulations of "true" wind field proposed by Testud and Chong (1983) were used to compare filtering effects and accuracy of the different methods: Dmsplines, bicubic splines, and bilinear elements.

Each simulation consists in:

- Considering a "true" wind field $F^{t}=\left(\Gamma^{t}, \Psi^{t}\right)$ due to a monochromatic wave $F^{t}=A_{0} \xi \exp \left(i \mathbf{k r}+\Phi_{0}\right)$, in which vector $\mathbf{r}$ determines the position of the observed point, $A_{0}$ the wave amplitude, $\xi$ a unit vector in the $\alpha$ plane defining the direction of wind perturbation, $\mathbf{k}$ the wave vector $(\lambda=2 \pi /|\mathbf{k}|$ being the wavelength), and $\Phi_{0}$ the phase.

- Defining the position of the two radars and the domain of interest in the $\alpha$-plane; the origin of coordinates is taken at the midpoint of $\bar{R}_{1} R_{2}$, the two radars being located $30 \mathrm{kms}$ apart, the $x$-axis is the axis $R_{1} R_{2}$ and $\Omega$ the rectangle $[-10 \mathrm{~km}, 10 \mathrm{~km}] \times[26 \mathrm{~km}, 46$ $\mathrm{km}]$.

- Simulating the samplings from the two radars, by assuming that they are distributed regularly in azimuth and radial distance (the increments being $0.7^{\circ}$ and 0.4 $\mathrm{km}$, respectively), and then adding to each value of the "true" field a random number simulating the radar noise, with rms value equal to $0.5 \mathrm{~m} \mathrm{~s}^{-1}$.

- Calculating filtered radial velocities $v_{1}$ and $v_{2}$, as solutions to two independent minimization problems (2.2).

- Recovering the filtered estimates $\Gamma^{f}$ and $\Psi^{f}$ of the field by using equations (2.1).

- Calculating the amplitude $A_{1}$ and phase $\Phi_{1}$ of the monochromatic wave having wave vector $\mathbf{k}$ and wind direction $\xi$, that best fits, in the least square sense, the output wind field $\left(\Gamma^{f}, \Psi^{f}\right)$, since the true wind field $\left(\Gamma^{t}, \Psi^{t}\right)$ is due to a monochromatic wave.

This process was repeated for $\lambda=0.5,1,1.5,2.5,3$, $3.5,4,5,6,7,8,10,15,20 \mathrm{~km}$, and for the angles $(\xi$, $O x)$ and $(\mathbf{k}, O x)$ equal to $0^{\circ}, 45^{\circ}, 90^{\circ}$.
Different criteria are used to compare the approximation methods:

- the ratio $A_{1} / A_{0}$, which measures the amplitude gain factor of the considered analysis. The experimental gain curve is obtained by connecting the ratio $A_{1} / A_{0}$ for the different values of $\lambda$.

- the phase shift $\left|\Phi_{1}-\Phi_{0}\right|$ introduced by the analysis.

- the residual noise after filtering that is defined by the deviations between the two components $\Gamma^{f}$ and $\Psi^{f}$ of the filtered field and the field associated to the best fitted wave $\left(A_{1}, \Phi_{1}, \mathbf{k}, \xi\right)$. For each component $\Gamma$ and $\Psi$, the rms values of these deviations are calculated on the interior points of the mesh used to define rectangular elements. A comparison of the true and the filtered divergence fields is carried out the same way.

\section{c. Calculation of filtered values}

In an $\alpha$-plane, the sample size is nearly 2500 points for each radar. In the finite element method, we define on $\Omega$ a rectangular grid with parallel directions to the $x$-axis and the $y$-axis, and with the grid spacing $\Delta x$ $=\Delta y=1 \mathrm{~km}$. The net is thus $21 \times 21$ points.

The smoothing parameter $\mu$ of (2.2) is adjusted so as to suppress the motions with subgrid scales. When the domain $\Omega$ is $R^{2}$, the amplitude gain function of the continuous filter associated to $J_{m}$ is $\Theta(\lambda)=1 /\left(1+\mu_{t}(2 \pi)\right.$ $\lambda)^{2 m}$ ) (Testud and Chong, 1983). The parameter $\mu_{t}$ controls the cutoff wavelength $\lambda_{c}\left[\Theta\left(\lambda_{c}\right)=0.5\right]$. We have verified experimentally that the parameter $\mu$ of the discrete filter verifies $\mu=\mu_{t} / S$ for cutoff wavelengths at least twice as large as the grid increment (where $S$ is the area of $\Omega$ ).

The characteristics of the three methods used for the numerical computations are listed:

\section{1) ANALYSIS A}

This analysis was used by Testud and Chong (1983) to filter dual Doppler radar data. The quadratic functional minimized is

$$
I(v)=\frac{1}{N} \sum_{i=1}^{N}\left[v\left(t_{i}\right)-u_{i}\right]^{2}+\mu J_{2}(v) .
$$

The approximation is made by using bilinear elements (nonconforming in the case of second order problems), and the discrete form of the variational problem is calculated by using finite differences.

The solution is obtained by solving $H x=p$, in which matrix $H$ of order 400 is symmetric positive and sparse (only 13 terms non-null on each line), using the conjugate gradient algorithm, which is very efficient in this type of problem. But as the approximate solution is not differentiable, it is necessary to estimate the divergence field $a$ posterior $i$ by a cubic spline differentiation procedure. The whole process is quite fast and, for each $\alpha$-plane, needs only $20 \mathrm{~s}$ of CPU time on the IRIS 80 computer. 


\section{2) Analyses B AND B'}

The approximation is made by using bicubic splines. The bicubic splines being twice continuously differentiable (see appendix A), they are admissible for $J_{2}$ (analysis $\mathrm{B}^{\prime}$ ) and $J_{3}$ (analysis B). In the two variants, the solution is obtained by solving $\mathbf{M} x=b$, in which $\mathbf{M}$ is a 7-banded symmetric positive matrix, each band containing 7 non-null terms (see Appendix B). As was the case for bilinear elements, the conjugate gradient algorithm is very efficient. The divergence field is obtained directly by differentiating the solution analytically. The results obtained in analyses $B$ and $\mathbf{B}^{\prime}$ are quite similar, though a little better in analysis B (Fig. 1 ), so in the following, only analysis B is compared to other methods.

Since $m$ controls the steepness of gain curve, the cutoff is sharper in analysis B.

The computing time is the same for $\mathbf{B}$ and $\mathbf{B}^{\prime}$, and varies from $50 \mathrm{~s}$ to $80 \mathrm{~s}$, in each $\alpha$-plane, depending on the values of smoothing parameter $\mu$, since the efficiency of the conjugate gradient increases proportionally to this parameter.

\section{3) ANALyses C AND C}

The approximation is made by using pseudocubic $\mathrm{C}$ and thin-plate splines $\mathrm{C}^{\prime}$. Since there are a great amount of data, we have subdivided the domain $\Omega$ into 36 rectangles, each containing 120 points maximum. (To fit the pieces smoothly together, it is necessary not to reduce the intersection of two "adjacent" rectangles to an edge but to have the intersection itself in the form of a rectangle.) In each rectangle, we obtain the solution by solving a system of $N$ linear equations $(N<120)$. We then fit the different pieces together with a continuously differentiable weighting function (Utreras, 1979).

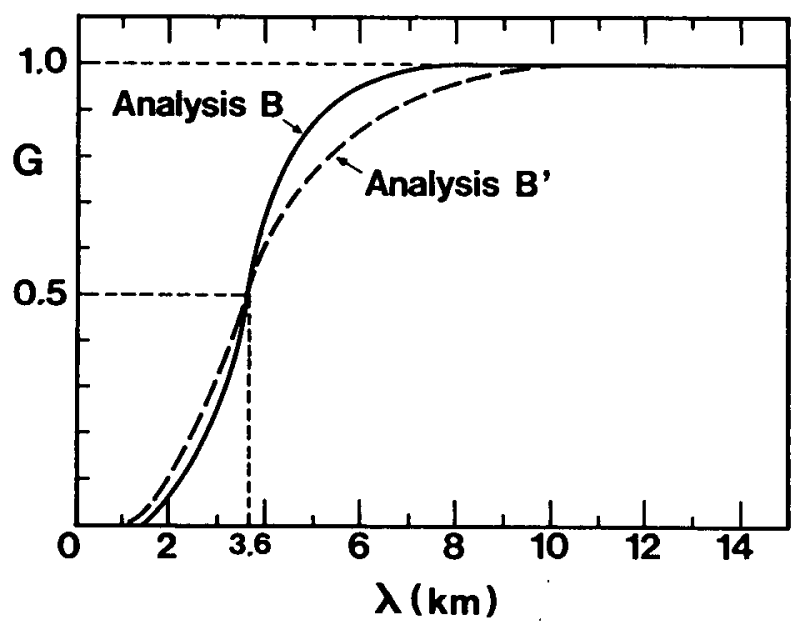

FIG. 1. Amplitude gain of the filtering as a function of the wavelength $\lambda$ obtained by bicubic spline approximation. Analysis $B$ is done in the case of functional $J_{3}$, analysis $\mathrm{B}^{\prime}$ in the case of $J_{2}$.

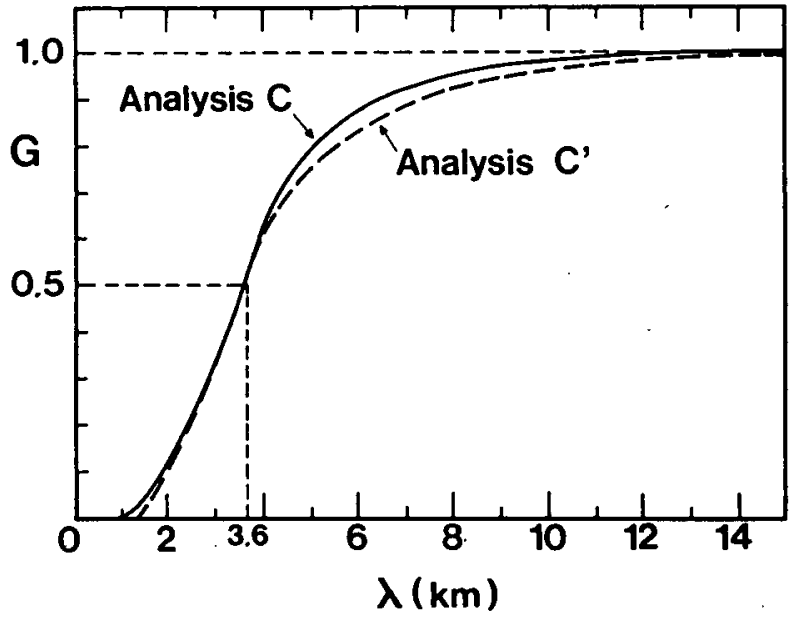

FiG. 2. Amplitude gain obtained by homogeneous splines. Analysis $C$ is carried out by using pseudocubic splines; analysis $C^{\prime}$, by using thin-plate splines.

Here too, the results obtained with the two variants are quite similar (Fig. 2). For the comparison with other methods only analysis $\mathrm{C}$ has been presented, because it is, in theory, more accurate to estimate the divergence field.

In this case, the computing time is nearly $15 \mathrm{~mm}$ for each $\alpha$-plane. It depends neither on the smoothing parameter, nor on the variant.

\section{d. Results of the simulation}

For analyses A, B and C we have verified:

- The linearity of the analyses. The variational analysis is linear for radial velocities, and obviously (because of the relations existing between Cartesian and radial fields) the whole process is a linear function of input data.

The experimental gain curves $G(\lambda)$ are independent of signal-to-noise ratio (SNR), and are very close to the theoretical gain curve $\Theta(\lambda)$ of the continuous lowpass filter applied directly to radial velocities.

- The isotropy of the analyses. The gain curve $C$ is unaffected by the direction of $\mathbf{k}$ in the $\alpha$-plane, since the basis functions are rotation invariant. On the gain curve $B$, there is a slight divergence in the case of nearly subgrid motions (around $2 \mathrm{~km}$ ). It is the same for analysis $\mathrm{A}$. In both cases, the basis functions have two privileged directions.

- The quasi-superposition of divergence gain curves and wind field gain curves.

- The negligibility of phase-shifts in all the analyses $\left(<1^{\circ}\right)$.

\section{e. Comparison of gain curves and error functions}

The filtering characteristics of analysis A (bilinear elements), B (bicubic splines), and $C$ (pseudocubic splines) can be compared with gain curves $G(\lambda)=A_{1} /$ 
$A_{0}$ (Fig. 3). They are taken for SNR fixed to 50 . The smoothing parameters have been chosen so that the three curves A, B and, C, intersect $G=0.5$ for $\lambda_{c}$ $=3.6 \mathrm{~km}$.

The cutoff around $\lambda_{c}$ is more progressive in $A$, and even more progressive in $\mathrm{C}$, than it is in $\mathrm{B}$. The slight superiority of analysis $\mathrm{B}$ over analysis A results from the minimization of $J_{3}$ rather than $J_{2}$, and from the basis that bicubic functions are better than bilinear functions to approximate cosine functions. Pseudocubic splines are not as satisfactory because the smoothing parameter and the filtering characteristics are fitted to the rectangular elements and to the grid scale.

The gain curve is an approximation of the filter response function since the data points are irregularly spaced. The spectrum of the output field contains other harmonics beyond the principle one as shown by Stephens and Polan (1971). The residual noise after filtering measures this discrepancy.

The rms values of the fluctuations in the filtered values $\Gamma^{f}$ and $\Psi^{f}$ around the best fitted wave $\Gamma^{0}, \Psi^{0}$ measured by $\operatorname{REF}\left(\Gamma^{0}\right)$ and $\operatorname{REF}\left(\Psi^{0}\right)$ are given in Fig. 4. The curves obtained for analyses $A, B$ and, $C$ have the same shape. The hump around $\lambda_{c}$ should be interpreted as due to a slight distortion for the range of wavelength located in the slope of the gain curve. For $\lambda \geqslant 10 \mathrm{~km}$ the curves are constant and the limiting values are principally due to the radar statistical error. When a noise-free field is simulated, the curves of the rms values have the same shape, except that the residual noise is the same for $\Gamma$ and $\Psi$ and the limiting values are negligible. These results corroborate the performance of the different analyses obtained by the gain curves. The same comments apply to the rms values of the fluctuations in the divergence field, measured by $\operatorname{REF}\left(\operatorname{div} \mathbf{F}^{0}\right.$ ).

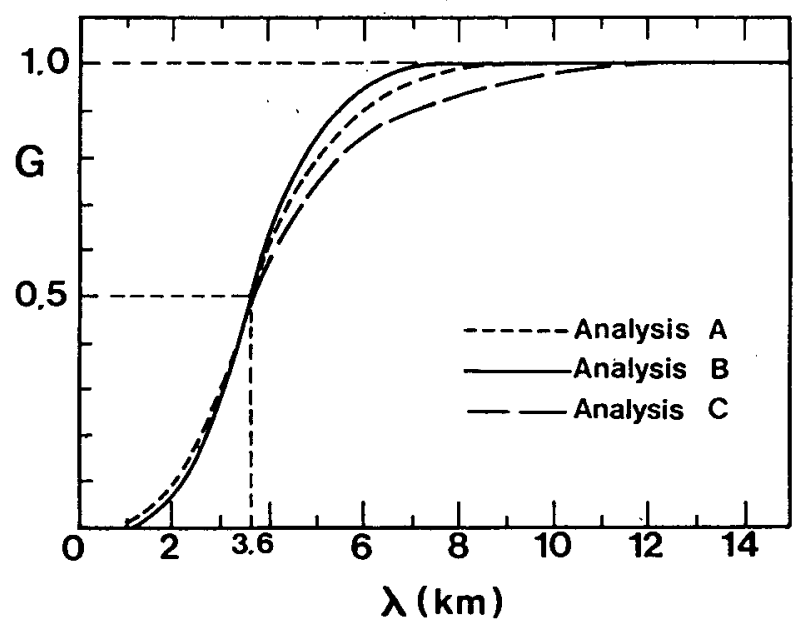

FIG. 3. Gain curves obtained by analyses A (bilinear approximation with functional $J_{2}$ ), B (bicubic spline approximation with $J_{3}$ ), and $\mathrm{C}$ (pseudocubic spline approximation).

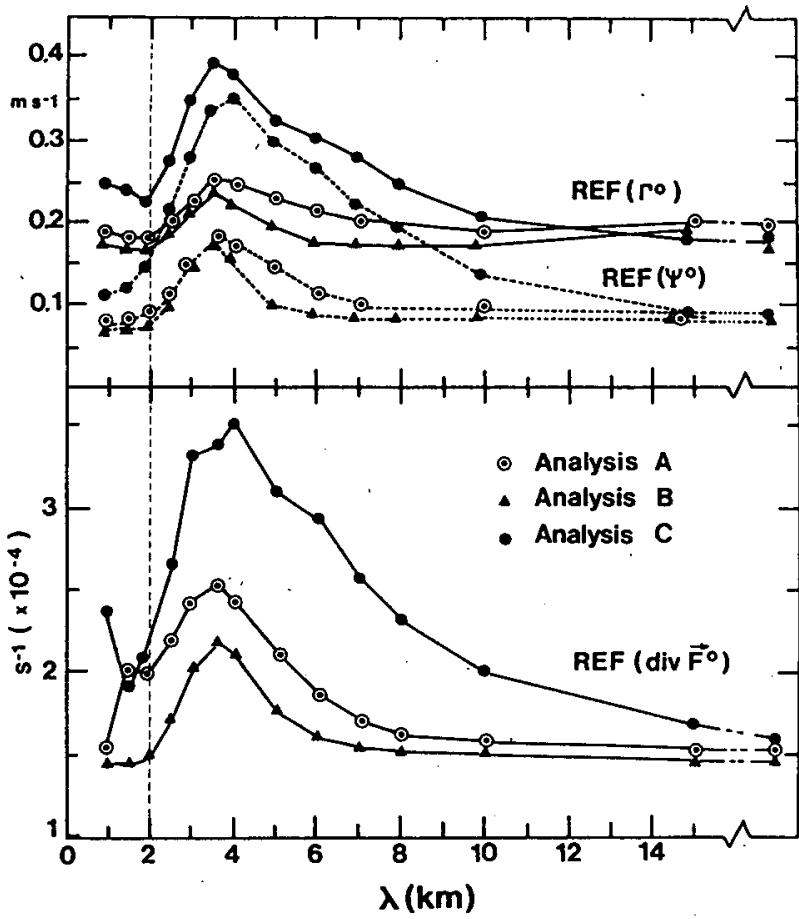

FIG. 4. Residual noise after filtering. Upper diagrams: rms values of random fluctuations in the filtered field $F^{0}=\left(\Gamma^{0}, \Psi^{0}\right)$. Lower diagram: the same but for div $\mathrm{F}^{0}$. The vertical dashed line indicates the minimum resolvable wavelength.

The best fitted wave has been calculated by using only the $19 \times 19$ interior points of the rectangular grid. If the boundary points are included, the gain curves remain unchanged but the rms values increase, particularly in the case of bilinear elements (analysis A). The same phenomenon can be observed when the domain of interest is closer to the radars' axis. As Testud and Chong (1983) pointed out, these effects are greater when the approximation of a 2-dimensional cosine function is carried out by a piecewise bilinear function.

\section{f. Effects of filtering parameters}

As the results obtained with bicubic splines are similar and only a little better than those obtained with bilinear elements, we can compare the influence of filtering parameters only for bicubic splines and pseudocubic splines.

Figure 5 represents the gain curves obtained for different values of the parameter $\mu$ with bicubic splines for the original grid, and for a grid with step $2 \mathrm{~km}$. Whereas for pseudocubic splines the low-pass filtering of the data depends only on the parameter $\mu$, for finite element methods the step of the grid is another filtering parameter. The least selective filter is obtained when $\mu=0$ (i.e., for classical least square fit), and it can be shown (Fig. 5) that in this case, the cutoff wavelength is around $1.2 \mathrm{~km}$ for a grid step of $1 \mathrm{~km}$, and $3.2 \mathrm{~km}$ for a step of $2 \mathrm{~km}$. This curve shows that there is an 


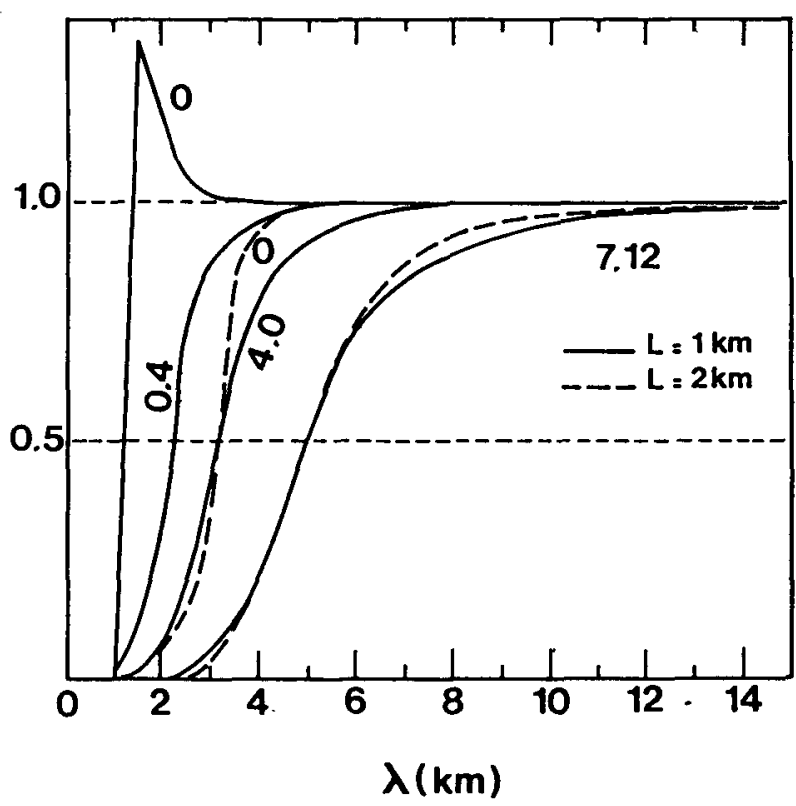

FIG. 5. Gain curves obtained for different values of the smoothing parameter $\mu$ and for two grid steps $L$.

amplification of the distortion for short waves. The weight function of the filter has sidelobes whose amplitude decreases rapidly with increasing smoothing parameter $\mu$. The choice of very small $\mu$ gives an unacceptable amplification of the shorter wavelengths and aliasing is very important in this case. To obtain a good variational filter, the parameter $\mu$ must be chosen so as to virtually eliminate the waves shorter than the minimum resolvable wavelength ( $2 \mathrm{~km}$ for a grid spacing of $1 \mathrm{~km}$ ).

If there are no additional specifications for the required passband, the parameter $\mu$ can be adjusted to minimize the mean square distance function between the true wind field and the filtered field, the summation being performed over the $19 \times 19$ interior grid points. For the grid step of $1 \mathrm{~km}$, the best results are obtained when the cutoff wavelength is around $4 \mathrm{~km}$, whereas for the step of $2 \mathrm{~km}$, the optimal cutoff wavelength is around $7 \mathrm{~km}$. Inversely, the grid step can be adjusted to the required filtering characteristics, provided that the sampling size is sufficient. For example, if the required cutoff wavelength is $5 \mathrm{~km}$, the grid spacing of $2 \mathrm{~km}$ is more accurate than that of $1 \mathrm{~km}$. Moreover, if the simulated samplings are made with increments twice as large in azimuth and radial distance, the grid step of $2 \mathrm{~km}$ fits better than that of $1 \mathrm{~km}$. The mean number of measurement points per grid mesh must exceed five. This restriction is made empirically in the case of this particular problem, and has no theoretical foundation. Since our sample contains a $P_{m-1}$ unisolvent subset, the smoothing problem has exactly one solution in $H^{m}(\Omega)$. The approximate solution is thus unique in any finite dimensional subspace, even if a rectangle of the grid happens not to contain a data point.

The pseudocubic splines enable one to reconstitute a larger bandpass, that depends on the sampling rate only. The optimum cutoff wavelength is around 1.8 $\mathrm{km}$. However, even in this case, the distance between the true and the filtered field is larger than the optimum distance obtained by bicubic splines. It appears that the reconstitution is better at the data points than at the mesh points (Wahba and Wendelberger, 1980).

\section{g. Discussion}

We have compared these methods with the conventional objective analysis by using the Cressman weighting function, where the influence radius $R=1.7$ $\mathrm{km}$ was adjusted to obtain the same cutoff wavelength $(3.6 \mathrm{~km})$. The cutoff around $\lambda_{c}$ is much more progressive than in analyses $\mathrm{A}, \mathrm{B}$ and, $\mathrm{C}$. The residual noise curves of the filtered field have a slighter hump and the limiting values are the same. In this case the distortion for medium waves $(2 \mathrm{~km} \leqslant \lambda \leqslant 6 \mathrm{~km})$ is smaller. However, the distance between the true field and the filtered field is greater than in analysis B, and the same phenomenon appears for the divergence field.

The bicubic splines, which are twice differentiable, are in theory better at approximating wind field and at estimating the divergence field in each $\alpha$-plane and might thus yield a better estimation of the third component when integrating the continuity equation. We simulated the sampling of a 3-dimensional nondivergent analytic field on $20 \alpha$-planes (for values of $\alpha$ varying from 0.5 to $19.5^{\circ}$ ). The specific form of the field is described in Chong et al. (1983). In each $\alpha$-plane the bidimensional field was filtered by using bicubic splines and the Cressman weighting function. We reconstituted the third component by upward integration (see Doviak et al., 1976). The rms errors $\sigma(W)$ which are listed in Table 1 for $\lambda_{H}=15 \mathrm{~km}, \lambda_{z}=25 \mathrm{~km}$, and for different values of $\alpha$, are smaller with bicubic splines than with the Cressman weighting function. However, in this particular problem, the filtered fields obtained by the different methods are quite similar, as expected, and any of these can be reliable.

TABLE 1. Root-mean-square values of the errors in the $w$ component within different planes.

\begin{tabular}{llllll}
\hline & $\alpha=0.5^{\circ}$ & $\alpha=5.5^{\circ}$ & $\alpha=10.5^{\circ}$ & $\alpha=15.5^{\circ}$ & $\alpha=19.5^{\circ}$ \\
\hline B-cubics & $0.14 \mathrm{~m} \mathrm{~s}^{-1}$ & $0.75 \mathrm{~m} \mathrm{~s}^{-1}$ & $1.98 \mathrm{~m} \mathrm{~s}^{-1}$ & $3.95 \mathrm{~m} \mathrm{~s}^{-1}$ & $6.85 \mathrm{~m} \mathrm{~s}^{-1}$ \\
Cressman & $0.18 \mathrm{~m} \mathrm{~s}^{-1}$ & $0.98 \mathrm{~m} \mathrm{~s}^{-1}$ & $2.50 \mathrm{~m} \mathrm{~s}^{-1}$ & $4.88 \mathrm{~m} \mathrm{~s}^{-1}$ & $8.50 \mathrm{~m} \mathrm{~s}^{-1}$ \\
\hline
\end{tabular}




\section{Conclusion}

The variational methods studied in this particular problem can be applied to a large class of problems and are very efficient, particularly if some improvements are provided:

- The dimension of the basis can be adjusted to the variation of the data by using an adaptative method to determine an optimal grid (with unequal steps), as was done by Dierckx (1981).

- The smoothing parameter can be chosen by the GCV method and depends, thus, on the amount of noise associated with the data.

- Different weights proportional to the variance of the measurement error can be associated with each observation.

- The variational form (1.3) can be modified so as to take into account other weak constraints.

For a small or a medium set of scattered data, homogeneous spline smoothing associated with the GCV method (Wahba, 1979) is an extremely efficient method. An approximate version with data compression of this method described by Wahba (1980) was successfully used for a large set of data by Hutchinson and Bischof (1983). For very large sets of data, rectangular elements, such as bicubic splines, are easy to compute and give accurate approximations.

\section{APPENDIX A}

\section{Cubic B-Splines}

Natural cubic splines have the smoothest interpolation property. Over all twice differentiable functions $f$ interpolating a given set of data, the interpolating spline minimizes:

$$
\int_{a}^{b}\left(f^{\prime \prime}(t)\right) d t
$$

The natural cubic spline with knots $\left\{x_{i}\right\}_{i=1}{ }^{n}$ can be thus written in the truncated power basis (which is a fundamental solution of iterated Laplacian operator $\Delta^{2}$ in one dimension):

$$
s(x)=d+e x+\sum_{i=1}^{n} c_{i}\left(x-x_{i}\right)_{+}{ }^{3} / 6
$$

where

$$
\left(x-x_{i}\right)_{+}{ }^{\alpha}= \begin{cases}\left(x-x_{i}\right)^{\alpha}, & \text { if } x \geqslant x_{i} \\ 0 & \text { elsewhere. }\end{cases}
$$

The cubic splines are piecewise cubic polynomial functions that are continuous and twice continuously differentiable over $[a, b]$.

The cubic B-spline basis, which has minimal support, is defined as the fourth divided-difference of truncated power basis, and then each $B_{i, 4}(x)$ is null outside the interval $\left[x_{i}, x_{i+4}\right]$. The B-spline functions can be cal- culated efficiently by using the following recurrence scheme:

$$
\left\{\begin{aligned}
& B_{i, 1}(x)= \begin{cases}1 & x_{i} \leqslant x<x_{i+1} \\
0 & \text { elsewhere }\end{cases} \\
& B_{i, k}(x)= \frac{\left(x-x_{i}\right)}{\left(x_{i+k-1}-x_{i}\right)} B_{i, k-1}(x) \\
& \quad+\frac{\left(x_{i+k}-x\right)}{\left(x_{i+k}-x_{i+1}\right)} B_{i+1, k-1}(x) .
\end{aligned}\right.
$$

When the points $\left\{x_{i}\right\}_{i=1}^{n}$ are spaced equally, Bsplines are shift-invariant (Fig. 6) and:

$B_{i, 4}(x)$

$$
=\left\{\begin{array}{rr}
1 / 6\left(1-3 z+3 z^{2}-z^{3}\right), & \\
\text { if } x \in\left[x_{i}, x_{i+1}\right], & z=\left(x_{i+1}-x\right) / h \\
2 / 3\left(-z^{2}+1 / 3 z^{3}\right) . & \\
\text { if } x \in\left[x_{i+1}, x_{i+2}\right], & z=\left(x_{i+2}-x\right) / h \\
1 / 2\left(1 / 3+z+z^{2}-z^{3}\right), & \\
\text { if } x \in\left[x_{i+2}, x_{i+3}\right], & z=\left(x_{i+3}-x\right) / h \\
z^{3} / 6, & \\
\text { if } x \in\left[x_{i+3}, x_{i+4}\right], & z=\left(x_{i+4}-x\right) / h .
\end{array}\right.
$$

To define the cubic B-spline basis, $n+6$ knots are necessary. It is usual to add 3 initial knots $x_{-2}, x_{-1}, x_{0}$ and 3 final knots $x_{n+1}, x_{n+2}, x_{n+3}$ (see Fig. 6). With this augmented set of knots, a cubic spline as a unique representation

$$
s(x)=\sum_{i=-2}^{n-1} a_{i} B_{i, 4}(x) \quad \text { or } \quad s(x)=\sum_{j=1}^{m} a_{j} B_{j, 4}(x)
$$
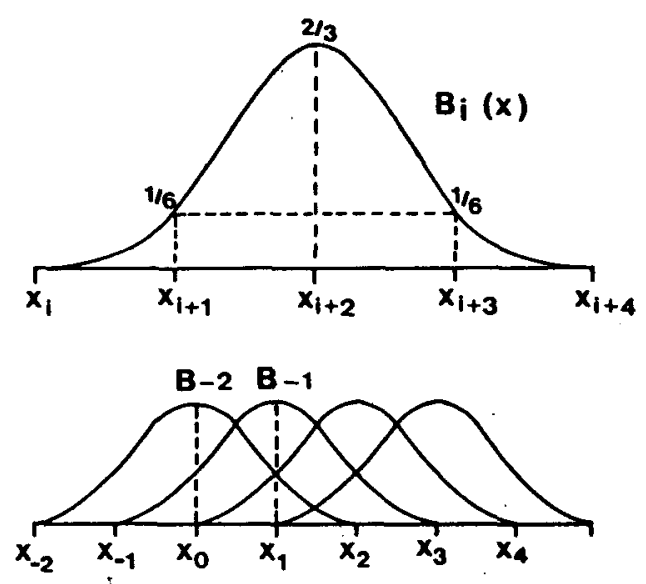

Fig. 6. B-spline basis corresponding to equally spaced knots. 
if the knots $x_{-2}, \ldots, x_{n+3}$ are renumbered $x_{1}, \ldots x_{m+4}$ for simplicity $(m=n+2)$.

\section{APPENDIX B}

\section{Assembling the Energy Matrix}

The minimum of $I(v)=1 / N \sum_{i=1}^{N}\left[v\left(t_{i}\right)-z_{i}\right]^{2}+\mu J_{3}(v)$ is sought in the subspace $V_{n}$ of $V$ spanned by $n=n_{x} n_{y}$ bicubic B-splines $v_{i}$. The minimum of $I(v)$ is the solution of the normal equation (1.13). The energy matrix $M$ is quite simple to compute. The element $m_{i j}$ is:

with

$$
m_{i j}=\frac{1}{N} \sum_{k=1}^{N} v_{i}\left(t_{k}\right) v_{j}\left(t_{k}\right)+\mu\left(v_{i}, v_{j}\right)_{3}
$$

$\left(v_{i}, v_{j}\right)_{3}=\sum_{\alpha=0}^{3} \frac{3 !}{\alpha !(3-\alpha) !} \int\left(\frac{\partial^{3}\left(v_{i}\right)}{\partial x^{\alpha} \partial y^{3-\alpha}}\right)\left(\frac{\partial^{3} v_{j}}{\partial x^{\alpha} \partial y^{3-\alpha}}\right)$.

The rectangular mesh is defined by the nodes $\left(x_{p}\right.$, $\left.y_{q}\right) 1 \leqslant p \leqslant n_{x}+4$ and $1 \leqslant q \leqslant n_{y}+4$. The basis functions are defined by $v_{i}(x, y)=B_{p}(x) B_{q}(y)$ where $i$ $=q+(p-1) n_{y}, 1 \leqslant p \leqslant n_{x}$ and $1 \leqslant q \leqslant n_{y}$. We note $B_{p}(x)$ instead of $B_{p, 4}(x)$.

As the products $B_{p}(x) B_{r}(x)$ are non-null if their supports intersect, i.e. if $|p-r|<4$, the inner product matrix $M$ is a seven banded matrix of seven terms each, symmetric positive definite. Even more, each band is also symmetric: this second symmetry comes from the product form of the basis $\left\{v_{i}\right\}_{i=1}{ }^{n}$. Owing to all these properties, the matrix $M$ is stored in 16 vectors of dimension $n$ containing $m_{i, i+g}$ for $g=0$ to $3, n_{y}$ to $n_{y}$ $+3,2 n_{y}$ to $2 n_{y}+3,3 n_{y}$ to $3 n_{y}+3$.

Let $\left(x_{k}, y_{k}\right)$ be the Cartesian coordinates of the data point $t_{k}$, then the first term of the element $m_{i j}$ (B.1) is:

$$
b_{i j}=1 / N \sum_{k=1}^{N} B_{p}\left(x_{k}\right) B_{q}\left(y_{k}\right) B_{r}\left(x_{k}\right) B_{s}\left(y_{k}\right),
$$

and the $i$ element of the load vector is

$$
f_{i}=1 / N \sum_{k=1}^{N} B_{p}\left(x_{k}\right) B_{q}\left(y_{k}\right) z_{k}
$$

These two terms can be calculated very quickly by a systematic algorithm. The trick is to compute, not one inner product after another, but the contributions to all the inner products from one point after another. This means that for one point $t_{k}$, we localize the rectangle $\left[x_{p}, x_{p+1}\right] \times\left[y_{q}, y_{q+1}\right]$ containing $t_{k}$, and compute the values $B_{d}\left(x_{k}\right), p-3 \leqslant d \leqslant p$ (resp. $B_{m}\left(y_{k}\right), q-3$ $\leqslant m \leqslant q$ ), since on a given interval, there are only four nonzero B-splines. We then compute the 16 products $B_{d}\left(x_{k}\right) B_{m}\left(y_{k}\right)$ with indexes $i_{d m}=m+(d-1) n_{y}$. To the 16 components $i_{d m}$ of the load vector, are added the associated product, and, for each line $i_{d m}$ of the matrix $M$, the corresponding 16 terms are also modified. Practically speaking, because of symmetries on the line $i_{d m}$, only the terms of indexes $j_{r s}$ with $d \leqslant r$ $\leqslant d+2$ and $m \leqslant s \leqslant m+2$ are modified.

The second term $c_{i j}$ of (B.1), corresponding to the contribution of $J_{3}$ is

$$
\begin{aligned}
c_{i j}= & \int\left(B_{p}^{\prime \prime \prime} B_{r}^{\prime \prime \prime}\right) d x \int\left(B_{q} B_{s}\right) d y \\
& +3 \int\left(B_{p}^{\prime \prime} B_{r}^{\prime \prime}\right) d x \int\left(B_{q}^{\prime} B_{s}^{\prime}\right) d y \\
& +3 \int\left(B_{p}^{\prime} B_{r}^{\prime}\right) d x \int\left(B_{q}^{\prime \prime} B_{s}^{\prime \prime}\right) d y \\
& +\int\left(B_{p} B_{r}\right) d x \int\left(B_{q}^{\prime \prime \prime} B_{s}^{\prime \prime \prime}\right) d y .
\end{aligned}
$$

Since the grid increment $h$ is the same in the two directions, and because of the symmetry and shift-invariance of B-splines, the quantities $\int_{0}^{4 h}\left(B_{0}{ }^{(\alpha)} B_{j}^{(\alpha)}\right)$ for

\begin{tabular}{|c|c|c|c|c|c|c|c|}
\hline \multicolumn{4}{|c|}{$c_{i, i+g} g=0$ to 3} & \multicolumn{4}{|c|}{$c_{i, i+g} g=n_{y}$ to $n_{y}+3$} \\
\hline 1880 & 181 & 184 & 263 & -181 & 167 & 549 & 37 \\
\hline$\overline{63}$ & $\overline{28}$ & $\overline{105}$ & $-\overline{1260}$ & $-\overline{28}$ & $-\overline{28}$ & $\overline{280}$ & $\overline{140}$ \\
\hline \multicolumn{4}{|c|}{$c_{i, i+g} g=2 n_{y}$ to $2 n_{y}+3$} & \multicolumn{4}{|c|}{$c_{i, i+g} g=3 n_{y}$ to $3 n_{y}+3$} \\
\hline 184 & 549 & 2 & 103 & 263 & 37 & 103 & 11 \\
\hline$\overline{105}$ & $\overline{280}$ & $\overline{7}$ & $\overline{840}$ & $\overline{1260}$ & $\overline{140}$ & $-\overline{840}$ & $\overline{1260}$ \\
\hline
\end{tabular}
$j=1$ to 4 and $\alpha=0$ to 3 are sufficient to determine all terms $c_{i j}$-after some laborious hours of hand computation. All the lines are identical (when translated) except near the edges where more terms are null. These coefficients are given below (the values had to be divided by $h^{4}$ ).

The same calculations were made when $c_{i j}$ was the contribution of $J_{2}$ instead of $J_{3}$ (the values in this case had to be divided by $h^{2}$ ). 


\begin{tabular}{|c|c|c|c|c|c|c|c|}
\hline \multicolumn{4}{|c|}{$c_{i, i+g} g=0$ to 3} & \multicolumn{4}{|c|}{$c_{i, i+g} g=n_{y}$ to $n_{y}+3$} \\
\hline 3256 & 23 & 64 & 131 & 23 & 752 & 1 & 83 \\
\hline$\overline{945}$ & $\overline{90}$ & $\overline{315}$ & $\overline{1890}$ & $\overline{90}$ & $\overline{1120}$ & $\overline{70}$ & $\overline{2016}$ \\
\hline \multicolumn{4}{|c|}{$c_{i, i+g} g=2 n_{y}$ to $2 n_{y}+3$} & \multicolumn{4}{|c|}{$c_{i, i+g} g=3 n_{y}$ to $3 n_{y}+3$} \\
\hline 64 & 1 & 2 & 23 & 131 & 83 & 23 & 31 \\
\hline$\overline{315}$ & $\overline{70}$ & $\overline{25}$ & $\overline{3150}$ & $\overline{1890}$ & $\overrightarrow{2016}$ & $\overline{3150}$ & $\overline{151200}$ \\
\hline
\end{tabular}

\section{REFERENCES}

Bates, D., and G. Wahba, 1983: A truncated singular value decomposition and other methods for generalized cross-validation. Tech. Rep. 715, University of Wisconsin, Madison, Statistics Department, 1-16.

Chong, M., J. Testud and F. Roux, 1893: Three-dimensional wind field analysis from dual doppler radar data. Part II: Minimizing the error due to temporal variation. J. Climate Appl. Meteor., 22, 1216-1226.

de Boor, C., 1978: A Practical Guide to Splines. Springer Verlag, 129-150.

Dierckx, P., 1981: An algorithm for surface-fitting with spline functions. IMA J. Numer. Anal. 1, 267-283.

Doviak, R., P. Ray, R. Strauch and L. Miller, 1976: Error estimation in wind fields derived from dual-Doppler radar measurements. J. Appl. Meteor., 15, 868-878.

Duchon, J., 1977: Splines minimizing rotation-invariant seminorms in Sobolev Spaces. Constructive Theory of Functions of Several Variables, W. Schempp and K. Zeller, Eds., Springer Verlag, 85-100.

Harder, R., and R. Desmarais, 1972: Interpolating using surfaces splines. J. Aircr., 9, 189-191.

Hutchinson, M., and R. Bischof, 1983: A new method for estimating the spacial distribution of the mean seasonal and annual rainfall applied to the Hunter Valley, New South Wales. Aust. Meteor. Mag., 31, 179-184.
Meinguet, J., 1979: Multivariate interpolation at arbitrary data points made simple. J. Appl. Math. Phys., 30, 292-304.

Sasaki, Y., 1971: A theoretical interpretation of anisotropically weighted smoothing on the basis of the numerical variational analysis. Mon. Wea. Rev., 99, 698-707.

Stephens, J. J., and A. L. Polan, 1971: Spectral modification by objective analysis. Mon. Wea. Rev., 99, 374-378.

Strang, G., and G. Fix, 1973: An Analysis of the Finite Element Method. Prentice Hall, 51-100.

Testud, J., and M. Chong, 1983: Three dimensional analysis from dual-Doppler radar data. J. Climate Appl. Meteor., 22, 12041215 .

Utreras, F., 1979: Cross validation techniques for smoothing spline functions in one or two dimensions. Smoothing Techniques for Curve Estimation, T. Gasser and M. Rosenblatt, Eds., Springer Verlag, 196-232.

Wahba, G., 1979: How to smooth curves and surfaces with splines and cross-validation. Proc. of the 24th Design of Experiments Conference, Madison, U.S. Army Research Office, 167-192.

- 1980: Splines bases, regularization, and generalized cross-validation for solving approximation problems with large quantities of noisy data. Approximation Theory III, W. Cheney, Ed., Academic Press, 905-912.

- and J. Wendelberger, 1980: Some new mathematical methods for variational objective analysis using splines and cross-validation. Mon. Wea. Rev., 108, 1122-1143. 